\title{
Mesenchymal stem cell-derived extracellular vesicles in therapy against fibrotic diseases
}

\author{
Yuling Huang and Lina Yang*
}

\begin{abstract}
Fibrosis is likely to occur in many tissues and organs to induce cicatrisation and dysfunction. The therapeutic regimens for delaying and even reversing fibrosis are quite limited at present. In nearly a decade, mesenchymal stem cells (MSCs) have been widely acknowledged as useful in treating fibrotic diseases in preclinical and clinical trials. Further preclinical studies indicated that the effects of mesenchymal stem cell-derived extracellular vesicles (MSC-EVs) are probably superior to that of MSCs. At present, MSC-EVs have attracted much attention in treating fibrosis of lung, liver, kidney, skin, and heart. By contrast, a significant knowledge-gap remains in treating fibrosis of other tissues and organs (including uterus, gastrointestinal tract, and peritoneum) with the aid of MSC-EVs. This review summarises the preclinical research status of MSC-EVs in treating fibrotic diseases and proposes solutions to existing problems, which contribute to further clinical research on the treatment of fibrotic diseases with MSC-EVs in the future.
\end{abstract}

Keywords: Fibrosis, Mesenchymal stem cells, Extracellular vesicles

\section{Introduction}

Fibrosis is regarded as the main cause of disability and death from many diseases. Fibrotic diseases may happen in various main human organs, including idiopathic pulmonary fibrosis (IPF), liver cirrhosis, renal fibrosis and cardiac fibrosis. Research shows that the mortality associated with organ fibrosis reaches nearly $50 \%$ in developed countries [1]. The difficulty in reversing fibrosis progress poses a challenge to treatment, which drives the emergence of new therapeutic methods. Numerous preclinical and clinical studies show that mesenchymal stem cells (MSCs) can relieve the progress of fibrosis of various organs [2]. Nevertheless, the mechanism of action underpinning the treatment of fibrotic diseases with mesenchymal stem cell-derived extracellular vesicles (MSC-EVs) remains under exploration. Thus, the review summarises preclinical studies on treating fibrotic

*Correspondence: yanglina1984@163.com

Departments of Geriatrics, The First Affiliated Hospital of China Medical University, 155th Nanjing North Street, Shenyang 110001, Liaoning, People's Republic of China diseases with MSC-EVs and proposes solutions to the existing problems.

\section{MSC-EVs}

MSCs, which are classified as multipotential stem cells, are widely available and can be separated from multiple tissues including bone marrow (bone marrow-derived MSCs (BMMSCs)), adipose tissues (adipose-derived MSCs (ADMSCs)), human umbilical cord (huMSCs), human placenta (hpMSCs), human liver (HLSCs), and menstrual blood (MenSCs). Previous studies suggested that MSCs function in target cells through differentiation and paracrine action; however, recent research shows that MSCs act in the treatment of various diseases mainly by excreting extracellular vesicles (EVs) and soluble nutritional factors [3]. At first, transplanted MSCs are directed to lesions through homing [4] and then deliver EVs and nutritional factors based on different methods, including forming tunnelling nanotubes and fusing with cells [5].

EVs can be divided into microvesicles (MVs), exosomes (Exos), and apoptotic bodies according to size, with respective diameters of $100-1000 \mathrm{~nm}, 30-150 \mathrm{~nm}$, and original author(s) and the source, provide a link to the Creative Commons licence, and indicate if changes were made. The images or other third party material in this article are included in the article's Creative Commons licence, unless indicated otherwise in a credit line to the material. If material is not included in the article's Creative Commons licence and your intended use is not permitted by statutory regulation or exceeds the permitted use, you will need to obtain permission directly from the copyright holder. To view a copy of this licence, visit http://creativecommons.org/licenses/by/4.0/. The Creative Commons Public Domain Dedication waiver (http://creativeco mmons.org/publicdomain/zero/1.0/) applies to the data made available in this article, unless otherwise stated in a credit line to the data. 
larger than $1000 \mathrm{~nm}$. Specific proteins, RNA, DNA, and lipids are packed into EVs during sorting [6]. Current research on EVs focuses on three aspects (Fig. 1): firstly, diseases are treated with EVs, for example, miR-150-5p in EVs from ADMSCs (ADMSC-EVs) relieves liver fibrosis by inhibiting the CXCL1 expression [7]. Secondly, EVs serve as a drug carrier: for example, MSC-EVs can be loaded with miR-101a showing an anti-fibrosis effect, thus mediating the cardioprotection with no need for direct intramuscular injection [8]. Thirdly, EVs act as a biomarker: for example, EVs can be used in diagnosis of various cancers and other diseases [9].

\section{Fibrosis and epithelial mesenchymal transition}

Fibrosis is actually a protective reaction required in the repair of damaged cellular tissue; however, fibrosis induces irreversible scarring and reduction in organ function when cellular tissue becomes severely damaged. The main changes of fibrosis include migration and proliferation of fibroblasts and accumulation of extracellular matrices, which damage the organ structure and functions [10]. The epithelial mesenchymal transition (EMT) is associated with the generation and development of fibrosis. EMT corresponds to a biological process during which epithelial cells are transformed into an interstitial cell phenotype through a specific procedure. Thereafter, epithelial cells lose their characteristics and are transformed into mesenchymal cells with loose structures. According to the differences between specific biological environments, EMT can be classified into three types, which are separately closely related to embryogenesis, tissue regeneration, and tumour invasion and metastasis (tissue regeneration forms the focus of the present discussion) [11].

\section{MSC-EVs and fibrotic diseases}

MSC-EVs transplantation, as an emerging and novel therapy, has been confirmed the benefits for various diseases, including fibrotic diseases. A clinical trial (NCT04173650), MSC EVs in Dystrophic Epidermolysis Bullosa, estimated study start date in April 2021; however, current research on fibrosis is limited to the preclinical stage. Many preclinical trials established different fibrotic models (Table 1). Due to various reasons leading to fibrosis, there are many methods to induce fibrosis models, such as chemical poisons. Cells treating with TGF- $\beta$ is a common way to induce EMT and fibrosis in most vitro trials. These preclinical studies have verified different MSC-EVs, especially exosomes, can exert antifibrosis effect through all kinds of mechanisms to treat fibrotic diseases. Additionally, miR-21/-23/-29/-let7 in MSC-EVs and TGF $\beta / S m a d$ signalling pathway are common molecular mechanism in treating fibrotic diseases, such as fibrosis of lung, liver, kidney, skin, and heart (Fig. 2).

\section{MSC-EVs and pulmonary fibrosis}

Pulmonary fibrosis (PF) is considered as a common manifestation of multiple lung diseases, such as some chronic diseases including IPF, silicosis, and pulmonary arterial hypertension [49]. Owing to the multiple underlying pathogenesis of PF and the lack of effective therapeutic drugs available clinically, developing new methods and drugs for preventing and treating PF has become the



Treatment

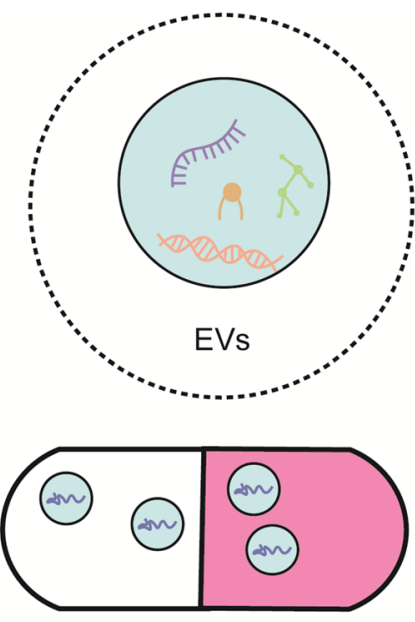

Drug carries

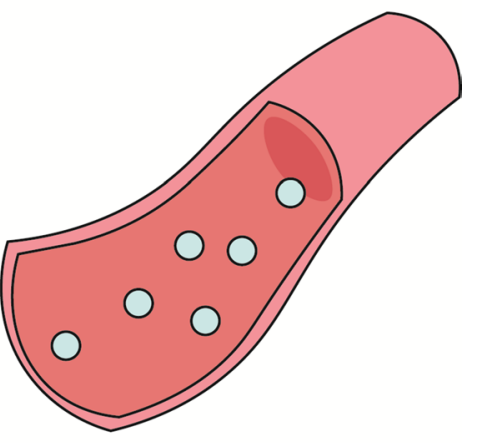

Biomarker

Fig. 1 Specific proteins, RNA, DNA, and lipids are packed into EVs. Current research on EVs focuses on three aspects, including EVs as a treatment, a drug carrier, and a biomarker. EVs, extracellular vesicles 


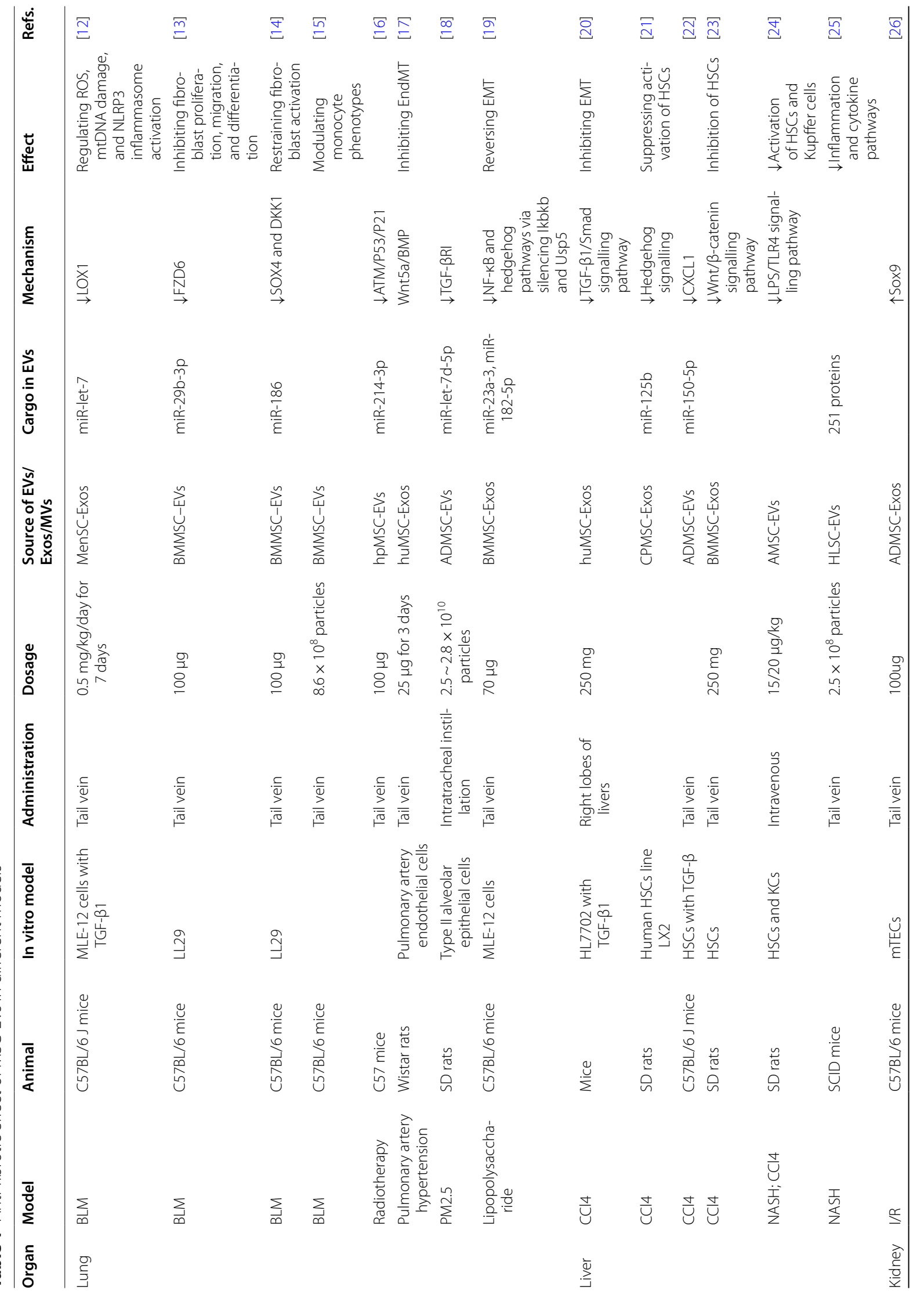









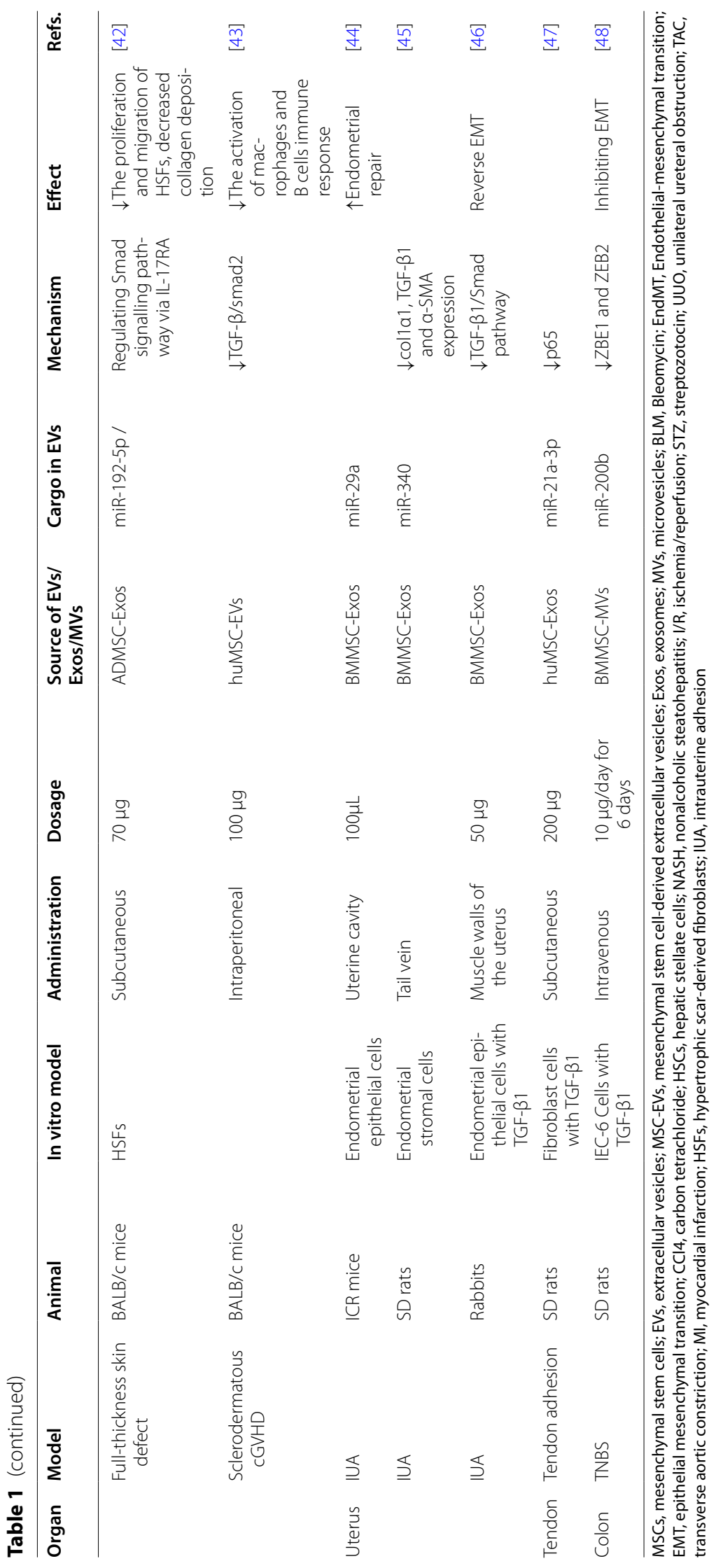




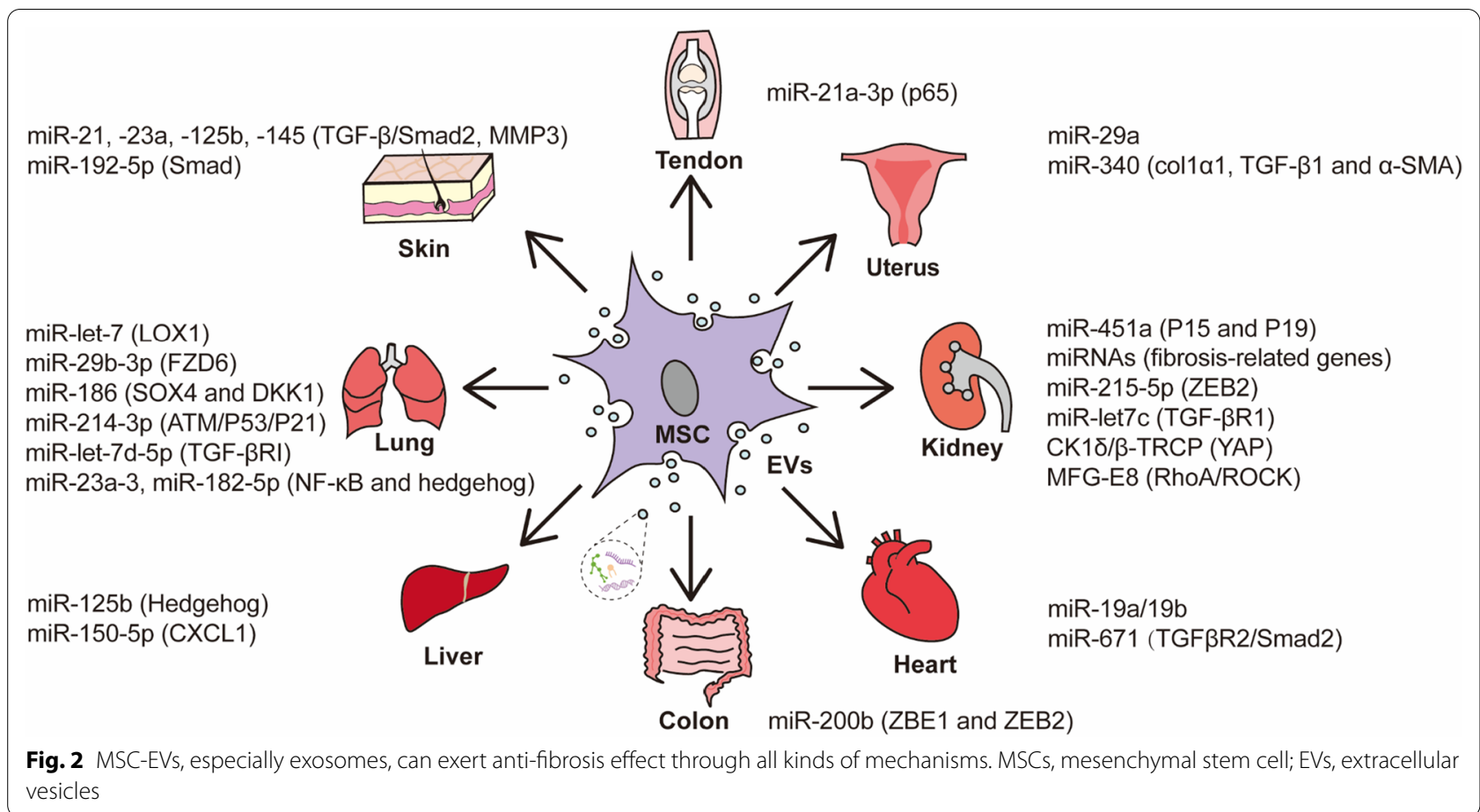

focus of many studies. MSCs have been verified to be effective in alleviating PF in clinical trials. Zhang et al. reported that an IPF patient subjected to long-term oxygen therapy exhibited improvements in physical performance, quality of life, and respiratory parameters after receiving huMSCs intravenous infusion over a followup period of 12 months [50]. PF is generally induced by intratracheal instillation of Bleomycin (BLM) in preclinical trials.

It should be pointed out that MSC-EVs as a potential antiviral therapy was reported in COVID-19 disease. Several clinical trials (NCT04276987, NCT04491240, and NCT04493242) are exploring the use of MSC-EVs in treating COVID-19 disease. Gentile et al. reviewed that intravenous infusion of MSCs played a significant role in counteracting fibrosis in individuals infected with SARSCoV-2 by the secreted EVs, especially exosomes [51]. In addition, ADMSCs and Stromal Vascular Fraction Cells (SVFs) improved lung fibrosis of COVID-19 patients through exosomal miRNAs [52].

The anti-fibrosis effect of miRNAs in MSC-EVs has become a research hotspot for PF induced by BLM. Sun et al. showed that exosomal miR-let-7 from MenSCs alleviates PF by regulating reactive oxygen species, mitochondrial DNA damage, and NLRP3 inflammasome activation [12]. Fibroblast proliferation is key to fibrosis. Wan et al. reported that BMMSC-EVs suppress fibroblast proliferation by down-regulating FZD6 expression in fibroblasts via miR-29b-3p [13]. Similarly, Zhou et al. found that miR-186 in BMMSC-EVs alleviates IPF by suppressing the expression of SOX4 and DKK1, thus blocking fibroblast activation [14]. Certainly, fibrosis is also closely correlated with inflammation. Mansouri et al. experimentally demonstrated that MSC-Exos prevent and reverse experimental PF by systematically modulating monocyte phenotypes [15].

MSC-EVs also ameliorate PF in other PF models. Chaubey et al. considered that TNF $\alpha$-stimulated gene- 6 (TSG-6) in huMSC-Exos play an important role in the hyperoxia-induced bronchopulmonary dysplasia model [53]. As for PF induced by intratracheal instillation of silica, the fibrosis gene, and inflammation are preliminarily explored, and mechanism still less studied. Phinney et al. further revealed that BMMSC-Exos reduce the extent of infiltration of monocytes and expression of profibrogenic genes (IL-10, and Col1 $\alpha 1$ ) in the lung, thus relieving PF [54]. MiR-214-3p in hpMSC-EVs attenuates radiationinduced DNA damage by down-regulating the ATM/ P53/P21 signalling pathway in PF induced by radiotherapy of the lung with a malignant tumour, thus relieving pulmonary inflammation and fibrosis [16]. Zhang et al. showed that huMSC-Exos block the progress of fibrosis by regulating the Wnt5a/BMP signalling pathway in pulmonary arterial hypertension models in vivo and in vitro [17]. Gao et al. found that ADMSC-EVs inhibit TGF- $\beta$ RI by transferring miR-let-7d-5p, further relieving PM2.5induced lung injury and PF [18]. Xiao et al. recently found that miR-23a-3 and miR-182-5p in BMMSC-Exos 
reverse the EMT process by blocking the activation of NF- $k B$ and hedgehog pathways via silencing of Ikbkb and destabilising IKK $\beta$ in lipopolysaccharide (LPS)-induced lung injury [19].

\section{MSC-EVs and liver fibrosis}

Liver fibrosis is triggered by chronic injury and inflammation, leading to liver cirrhosis, portal hypertension, and liver failure. At present, the therapy on liver cirrhosis with MSCs has realised remarkable achievements in clinical trials. A pilot four-phase clinical trial (NCT04243681) regarding MSCs infusion for treating decompensated cirrhosis has been completed in India. Numerous studies have been conducted on preclinical therapy of liver fibrosis with MSC-EVs.

It is common to induce liver fibrosis by using carbon tetrachloride $\left(\mathrm{CCl}_{4}\right)$ during preclinical trials. $\mathrm{Li}$ et al. suggested that huMSC-Exos alleviate liver fibrosis by inactivating the TGF- $\beta 1 /$ Smad signalling pathway and inhibiting EMT [20]. Hyun et al. showed that miR-125b in exosomes of chorionic plate-derived mesenchymal stem cells (CP-MSCs) drives the regression of fibrosis by inhibiting the activation of hedgehog signals [21]. Additionally, Du et al. proposed that miR-150-5p in ADMSCEVs can weaken $\mathrm{CCl}_{4}$-induced liver fibrosis by inhibiting the expression of CXCL1 [22]. Ohara et al. reported that EVs from amnion-derived MSCs (AMSC-EVs) ameliorate liver fibrosis by weakening the activation of hepatic stellate cells and Kupffer cells [24]. Rong et al. further proved that BMMSCs-Exos can alleviate $\mathrm{CCL}_{4}$-induced liver fibrosis by inhibiting the activation of hepatic stellate cells via the Wnt/ $\beta$-catenin pathway [23].MSC-EVs can also improve liver function to some extent in the other models for liver fibrosis. For example, Bruno et al. found that HLSC-derived EVs attenuate liver fibrosis and inflammation by regulating fibrosis genes in a murine model of non-alcoholic steatohepatitis (NASH) [25].

Exogenous modification, preconditioning, and the use of bio-gel prove to be effective in strengthening the antifibrosis effect of MSC-EVs in liver. MiR-122 modification can improve the therapeutic efficacy of ADMSCs via exosome-mediated miR-122 communication [55]. The miR-181-5p-bearing exosomes increase autophagy and reduce TGF- $\beta 1$-induced liver fibrosis by inhibiting the STAT3/Bcl-2/Beclin1 pathway [56]. Similarly, exosomes derived from mmu_circ_0000623-modified ADMSCs prevent liver fibrosis by activating autophagy [57]. The exosomes derived from miR-145-5p-modified huMSCs alleviate liver fibrosis by down-regulating the expression of actin-binding protein 1 [7]. The PEG hydrogels can prolong the bioavailability of MSC-EVs in targeted livers, thus enhancing the anti-fibrosis characteristics thereof [58]. Relative to MSC-EVs, MSC-EVs preconditioned with IFN- $\gamma$ better alleviate the inflammation and fibrosis of the murine model with liver cirrhosis [59].

\section{MSC-EVs and renal fibrosis}

Renal fibrosis is mainly induced by various chronic kidney diseases. It is considered the main pathological change and common pathway in the final stage of kidney disease, however, no targeted therapy yet exists to reverse renal fibrosis [60]. At present, research further verifies that MSC-EVs are the key to treating renal fibrosis models.

Multiple studies have shown that MSC-EVs can relieve renal fibrosis. MSC-EVs can inhibit the fibrotic transformation of various renal parenchyma cells at a cellular level. Zhu et al. believed that ADMSC-Exos block the transition of TGF- $\beta 1$-induced tubular epithelial cells to their profibrogenic phenotype [26]. Zhong et al. verified that huMSC-MVs reboots the cell cycle and reverses the EMT in vivo and in vitro through negative regulation of P15 and P19 via miR-451a [27]. Jin et al. found that ADMSC-Exos attenuate the EMT of podocytes by inhibiting ZEB2 via miR-215-5p [30]. MSC-EVs slow the progress of renal fibrosis by affecting fibrosis-related genes at a molecular level. Wang et al. showed that BMMSCs can suppress the expression of fibrosis genes by delivering exogenous miR-let-7c via exosomes [31]. Grange et al. revealed that MSC-EVs down-regulate fibrosis genes in a chronic renal injury model induced by diabetes mellitus [28]. Kholia et al. reported that MSC-EVs significantly down-regulate the fibrosis genes $\alpha$-SMA, TGF- $\beta 1$, and Col1a1 after their having been up-regulated by aristolochic acid [29]. In addition, MSC-EVs also alleviate renal fibrosis via various signalling pathways: Ji et al. found that huMSC-Exos attenuate renal fibrosis through CK1 $\delta / \beta$-TRCP-mediated YAP degradation in a UUO model [32]. Similarly, Liu et al. suggested that huMSCExos protect against renal interstitial fibrosis through the ROS-mediated P38MAPK/ERK signalling pathway [33]. Shi et al. found that BMMSC-EVs attenuate renal fibrosis, in part by inhibiting the RhoA/ROCK pathway [34].

Researchers have developed many methods with which to enhance the characteristics of MSC-EVs against renal fibrosis. Modification of MSC-EVs is taken as an approach to strengthen the anti-fibrotic nature thereof. Zhou et al. modified EVs via KMP2 to ameliorate chronic renal fibrosis in I/R mice [61]. Chen et al. suggested that GDNF-modified ADMSC-Exos alleviate renal fibrosis by activating the SIRT1/eNOS signalling pathway [62]. Also, Zhang et al. prolonged the release of EVs through use of RGD hydrogels [63]. The collagen matrices employed by Liu et al. exhibit similar characteristics [64]. Additionally, Zhang et al. revealed that the over-expression of Oct- 4 enhances the anti-fibrotic effect of MSC-EVs [65]. 


\section{MSC-EVs and cardiac fibrosis}

Myocardial infarction (MI) is regarded as one of the main causes of death from cardiovascular disease. The damaged cardiomyocytes are replaced with fibrous scars and cardiac remodelling leads to cardiac dysfunction [66]. Myocardial remodelling has long been a focus of research (post-MI). The amelioration of myocardial fibrosis with MSC-EVs has become a research hotspot.

Cardiac fibroblasts are crucial for myocardial fibrosis. Chen et al. found that BMMSC-Exos promotes the early senescence of myofibroblasts in vitro [35]. Ferguson et al. reported that MSC-Exos can inhibit the generation of type I collagen genes in primary human cardiac fibroblasts [67]. Additionally, MSC-EVs relieve cardiac remodelling by delivering diverse information. Wang et al. suggested that BMMSC-Exos can promote recovery of heart function via miR-19a/19b to decrease myocardial fibrosis in a MI model [36]. Deng et al. suggested that ADMSC-Exos alleviates myocardial injuries after MI by activating the S1P/SK1/S1PR1 signalling pathway and promoting macrophage M2 polarisation [37]. Recently, Wang et al. found that miR-671 in ADMSC-Exos alleviate myocardial injury via targeting TGF $\beta$ R2/Smad2 axis in vivo and vitro [38].

Like liver, kidney, and other organs, many methods can assist MSC-EVs in improving cardiac function and alleviating fibrosis, as shown in Table 2. MSC-EVs are parcelled in PGN hydrogels [68], alginate hydrogels [69], and (RADA) ${ }_{4}$-SDKP hydrogels [70] to prolong retention so that EVs can be stably and sustainably released. The modification and preconditioning of EVs also can reduce cardiac fibrosis. Pan et al. revealed that miR-146a-modified ADMSC-Exos inhibit the expression of EGR1 after transcription, which reverses acute myocardial infarction or hypoxia-induced TLR4/NFkB signal activation. The activation of TLR4/NFKB signals plays an important role in promoting cardiomyocyte apoptosis, inflammatory response, and fibrosis [71]. The exosomes engineered from IMTP can specifically target the ischemic myocardium [72]. FNDC5 or irisin increases the secretion of exosomes from BMMSCs [73]. Those exosomes with over-expressed HIF- $1 \alpha$ protect cardiac function by promoting neovascularisation and inhibiting fibrosis [74]. B2M-deficient huMSC-Exos inhibit cardiac fibrosis and restores cardiac function [75]. The ischemically preconditioned exosomes relieve fibrosis after MI through miR22 [76]. Hypoxia strengthens the activities of miR-210 and neutral sphingomyelinase 2 in MSC-Exos, thus playing a cardioprotective role [77].

\section{MSC-EVs and skin scars}

Skin scars are common and an effective therapeutic method for human scarless wound-healing remains to be determined. Fang et al. suggested that huMSC-Exos suppress myofibroblast differentiation by inhibiting the TGF- $\beta /$ Smad2 pathway during the wound-healing [39]. Wang et al. found that ADMSC-Exos reduce scar-formation by regulating the ratios of type III: type I collagens, TGF- $\beta 3$ : TGF- $\beta 1$, and MMP3: TIMP1 and fibroblast differentiation [40]. Dalirfardouei et al. thought that MenSC-Exos may alleviate scar formation by reducing the Col1: Col3 ratio [41]. ADMSC-Exos ameliorate fibrosis via the miR-192-5p/IL-17RA/Smad axis in hypertrophic scars [42]. HuMSC-EVs prevent fibrosis in the cGVHD mouse model with scleroderma by suppressing the activation of macrophages and $\mathrm{B}$ cell

Table 2 Methods to enhance the anti-fibrosis effect of MSC-EVs in different organs

\begin{tabular}{|c|c|c|c|}
\hline & Exogenous modification & Bio-gel & Preconditioning \\
\hline \multirow[t]{4}{*}{ Liver } & miR-122 [55] & PEG hydrogels [58] & IFN- $\gamma[59]$ \\
\hline & miR-181-5p [56] & & \\
\hline & mmu_circ_0000623 [57] & & \\
\hline & miR-145-5p [7] & & \\
\hline \multirow[t]{2}{*}{ Kidney } & KMP2 [61] & RGD hydrogels [63] & Oct-4 overexpression [65] \\
\hline & GDNF [62] & Collagen matrix [64] & \\
\hline \multirow[t]{5}{*}{ Heart } & miR-146a [71] & PGN hydrogels [68] & FNDC5 or irisin [73] \\
\hline & IMTP [72] & Alginate hydrogels [69] & HIF-1a [74] \\
\hline & & $(\mathrm{RADA})_{4}$-SDKP hydrogels [70] & B2M-deficient [75] \\
\hline & & & Ischemia [76] \\
\hline & & & Hypoxia [77] \\
\hline Skin & TSG-6 [78] & BSSPD hydrogels [79] & \\
\hline Tendon adhesion & & & Hydroxycamptothecin [80] \\
\hline Urethra & & & TNFa [81] \\
\hline
\end{tabular}

MSCs, mesenchymal stem cells; MSC-EVs, mesenchymal stem cell-derived extracellular vesicles 
immune response [43]. Jiang et al. suggested that TSG6-modified MSC-Exos attenuate collagen deposition and scar-formation during the wound-healing [78]. Additionally, miR-29b-3p secreted by BMMSC-EVs parcelled in BSSPD hydrogels inhibits the proliferation and migration of endothelial cells and fibroblasts and the expression of Col1A1 of fibroblasts by curbing the PI3K/Akt, Erk1/2, and Smad3/TGF- $\beta 1$ signalling pathways, thus realising scarless wound-healing [79].

\section{MSC-EVs and other fibrotic diseases}

Intrauterine adhesion (IUA), also called Asherman syndrome, triggered by endometrial injury is a common gynaecological disease induced by basement membrane injury in the endometrium and exposure of myometrial tissues. At present, IUA treatment mainly relies on hysteroscopic surgery to eliminate adhesions, in concert with hormonotherapy [82]. Tan, Xia, and Ying thought that miR-29a in BMMSCs-Exos may be an important factor affecting resistance to fibrosis during endometrial repair of IUA [44]. Xiao et al. found that BMMSC-Exos alleviate endometrial fibrosis by transferring miR-340 to endometrial stromal cells [45]. In addition, Yao et al. verified that BMMSC-Exos can reverse the EMT of rabbit endometrial epithelial cells through the TGF- $\beta 1 / \mathrm{Smad}$ pathway [46]. Tendon adhesion, a common complication of tendon injuries, influences the recovery of motor function. Yao et al. showed that huMSC-Exos are likely to regulate the p65 activity by delivering low-abundance miR-21a-3p, while showing anti-adhesion characteristics [47]. Li et al. revealed that hydroxycamptothecin-induced huMSC-EVs inhibit myofibroblast differentiation by activating the endoplasmic reticulum of fibroblasts and exhibit a strengthened anti-adhesion effect after Achilles tendon injury [80]. Moreover, exosomes can prevent the formation of urethral stricture by regulating fibrosis and angiogenesis, TNF $\alpha$-preconditioned huMSC-Exos are more effective in inhibiting urethra fibrosis and stricture via up-regulating miR-146a compared to untreated huMSC-Exos [81]. BMMSC-MVs can inhibit the EMT and further colonic fibrosis by targeting ZEB1 and ZEB2 in vivo and in vitro through miR-200b [48].

\section{Problems and prospects}

MSC-EVs exert a positive effect on various fibrotic diseases in the aforementioned research. The current research aiming at treatment of fibrosis with MSC-EVs is in its preclinical stage. There was a lack of large animal experiments in MSC-EVs treating fibrotic diseases; however, several studies all found that MSC-EVs can attenuate renal or myocardial fibrosis in the swine models of metabolic syndrome and renovascular disease by restoring the renal microcirculation and preserving renal cellular integrity [83-85]. Even in research on the treatment of peritoneal dialysis, MSCs for peritoneal fibrosis remain under exploration and MSC-EVs have rarely been involved in the research. Moreover, huMSCs relieve peritoneal fibrosis in rats induced by MGO by up-regulating miR-153-3p [86]. Numerous tests are still needed to support further clinical research in MSCs. Most fibrosis models have not been normalised for different tissues and organs. PF is mainly induced by using BLM and silica and the liver fibrosis is mainly triggered with $\mathrm{CCl}_{4}$ in animal experiments; it is possible to induce fibrosis of different tissues and organs with TGF- $\beta 1$ during the cell tests.

The dosage and administration route of MSC-EVs are still under exploration and the most effective administration has not yet been determined. A systematic review concluded that localised administration for delivering MSC-EVs was common in treating ophthalmic, skin and musculoskeletal conditions, whereas systemic administration was popular in autoimmune, brain, gastrointestinal, liver and pancreatic diseases [87]. Similar to MSCs, topical application of MSCEVs is the least invasive method; arterial injection and direct injection can reduce EVs loss; intravenous administration is relatively ease and safe [88].

Overall, this review summarises preclinical studies on application of MSC-EVs in treating various fibrotic diseases, including lung, liver, kidney, heart, skin, and endometrium. MSC-EVs are promising as drugs for treating fibrotic diseases in preclinical trials. More efforts are needed before further clinical application due to the limited research completed at time of writing.

\section{Abbreviations \\ MSCs: Mesenchymal stem cells; MSC-EVs: Mesenchymal stem cell-derived extracellular vesicles; IPF: Idiopathic pulmonary fibrosis; BMMSCs: Bone marrow-derived MSCs; ADMSCs: Adipose-derived MSCs; huMSCs: Human umbilical cord-derived MSCs; hpMSCs: Human placenta-derived MSCs; HLSCs: Human liver stem cells; MenSCs: Menstrual blood-derived stem cells; MVs: Microvesicles; Exos: Exosomes; ADMSC-EVs: EVs from ADMSCs; EMT: Epithelial mesenchymal transition; PF: Pulmonary fibrosis; BLM: Bleomycin; SVFs: Stromal Vascular Fraction Cells; MSC-CM: Conditioned medium of MSCs; HAECs: Human amniotic epithelial cells; TSG-6: TNF a-stimulated gene-6; CCl 4 : Carbon tetrachloride; CP-MSCs: Chorionic plate-derived mesenchymal stem cells; AMSC-EVs: EVs from amnion-derived MSCs; NASH: Non-alcoholic steato- hepatitis; UUO: Unilateral ureteral obstruction; MI: Myocardial infarction; IUA: Intrauterine adhesion.}

\section{Acknowledgements}

We are grateful to the National Natural Science Foundation of China (Grant No. 81970663) and the Natural Science Foundation of Liaoning Province (Grant No. 2019-BS-277).

\section{Authors' contributions}

Lina Yang contributed to the conception of this manuscript. Yuling Huang and Lina Yang drafted and revised the manuscript. Both authors read and approved the final manuscript. 


\section{Funding}

This work was supported by the National Natural Science Foundation of China (Grant No. 81970663) and the Natural Science Foundation of Liaoning Province (Grant No. 2019-BS-277).

\section{Availability of data and materials}

Not applicable.

\section{Declarations}

\section{Ethical approval and consent to participate}

Not applicable.

\section{Consent for publication}

Not applicable.

\section{Competing interests}

The authors report no relationships that could be construed as a conflict of interest.

Received: 1 June 2021 Accepted: 16 July 2021

Published online: 04 August 2021

\section{References}

1. Friedman SL, Sheppard D, Duffield JS, Violette S. Therapy for fibrotic diseases: nearing the starting line. Sci Transl Med. 2013;5(167).

2. El Agha E, Kramann R, Schneider RK, Li X, Seeger W, Humphreys $\mathrm{BD}$, et al. Mesenchymal stem cells in fibrotic disease. Cell Stem Cell. 2017;21(2):166-77.

3. Rangel EB, Gomes SA, Kanashiro-Takeuchi R, Saltzman RG, Wei C, Ruiz P et al. Kidney-derived c-kit(+) progenitor/stem cells contribute to podocyte recovery in a model of acute proteinuria. Sci Rep. 2018;8.

4. Zhang K, Chen S, Sun H, Wang L, Li H, Zhao J, et al. In vivotwo-photon microscopy reveals the contribution of Sox $9(+)$ cell to kidney regeneration in a mouse model with extracellular vesicle treatment. J Biol Chem. 2020;295(34):12203-13.

5. Zhao L, Hu C, Han F, Wang J, Chen J. Regenerative abilities of mesenchymal stem cells via acting as an ideal vehicle for subcellular component delivery in acute kidney injury. J Cell Mol Med. 2020;24(9):4882-91.

6. Gandham S, Su X, Wood J, Nocera AL, Alli SC, Milane L, et al. Technologies and standardization in research on extracellular vesicles. Trends Biotechnol. 2020;38(10):1066-98.

7. Zhang P, Gan Z, Tang L, Zhou L, Huang X, Wang J. Exosomes from microRNA-145-5p-modified HUCB-MSCs attenuate CCl4-induced hepatic fibrosis via down-regulating FSCN1 expression. Life Sci. 2021:119404-119404.

8. Wang J, Lee CJ, Deci MB, Jasiewicz N, Verma A, Canty JM et al. MiR-101a loaded extracellular nanovesicles as bioactive carriers for cardiac repair. Nanomed Nanotechnol Biol Med. 2020;27.

9. Shao H, Im H, Castro CM, Breakefield X, Weissleder R, Lee H. New technologies for analysis of extracellular vesicles. Chem Rev. 2018;118(4):1917-50.

10. Henderson NC, Rieder F, Wynn TA. Fibrosis: from mechanisms to medicines. Nature. 2020;587(7835):555-66.

11. Angela Nieto M, Huang RY-J, Jackson RA, Thiery JP. EMT: 2016. Cell. 2016;166(1):21-45.

12. Sun L, Zhu M, Feng W, Lin Y, Yin J, Jin J et al. Exosomal miRNA Let-7 from menstrual blood-derived endometrial stem cells alleviates pulmonary fibrosis through regulating mitochondrial DNA damage. Oxid Med Cell Longev. 2019;2019.

13. Wan X, Chen S, Fang Y, Zuo W, Cui J, Xie S. Mesenchymal stem cellderived extracellular vesicles suppress the fibroblast proliferation by downregulating FZD6 expression in fibroblasts via micrRNA-29b-3p in idiopathic pulmonary fibrosis. J Cell Physiol. 2020;235(11):8613-25.

14. Zhou J, Lin Y, Kang X, Liu Z, Zhang W, Xu F. microRNA-186 in extracellular vesicles from bone marrow mesenchymal stem cells alleviates idiopathic pulmonary fibrosis via interaction with SOX4 and DKK1. Stem Cell Res Therapy. 2021;12(1).

15. Mansouri N, Willis GR, Fernandez-Gonzalez A, Reis M, Nassiri S, Mitsialis SA et al. Mesenchymal stromal cell exosomes prevent and revert experimental pulmonary fibrosis through modulation of monocyte phenotypes. JCl Insight. 2019;4(21).

16. Lei X, He N, Zhu L, Zhou M, Zhang K, Wang C et al. Mesenchymal stem cell-derived extracellular vesicles attenuate radiation-induced lung injuryviamiRNA-214-3p. Antioxid Redox Signal. 2020.

17. Zhang Z, Ge L, Zhang S, Wang J, Jiang W, Xin Q, et al. The protective effects of MSC-EXO against pulmonary hypertension through regulating Wnt5a/BMP signalling pathway. J Cell Mol Med. 2020;24(23):13938-48.

18. Gao Y, Sun J, Dong C, Zhao M, Hu Y, Jin F. Extracellular vesicles derived from adipose mesenchymal stem cells alleviate PM2.5-induced lung injury and pulmonary fibrosis. Med Sci Monitor Int Med J Exp Clin Res. 2020;26:e922782.

19. Xiao K, He W, Guan W, Hou F, Yan P, Xu J, et al. Mesenchymal stem cells reverse EMT process through blocking the activation of NF-kappa $B$ and Hedgehog pathways in LPS-induced acute lung injury. Cell Death Disease. 2020;11(10).

20. Li T, Yan Y, Wang B, Qian H, Zhang X, Shen L, et al. Exosomes derived from human umbilical cord mesenchymal stem cells alleviate liver fibrosis. Stem Cells Dev. 2013;22(6):845-54.

21. Hyun J, Wang S, Kim J, Kim GJ, Jung Y. MicroRNA125b-mediated Hedgehog signaling influences liver regeneration by chorionic plate-derived mesenchymal stem cells. Sci Rep. 2015;5.

22. Du Z, Wu T, Liu L, Luo B, Wei C. Extracellular vesicles-derived miR-150-5p secreted by adipose-derived mesenchymal stem cells inhibits CXCL1 expression to attenuate hepatic fibrosis. J Cell Mol Med. 2020.

23. Rong X, Liu J, Yao X, Jiang T, Wang Y, Xie F. Human bone marrow mesenchymal stem cells-derived exosomes alleviate liver fibrosis through the Wnt/beta-catenin pathway. Stem Cell Res Therapy. 2019;10.

24. Ohara M, Ohnishi S, Hosono H, Yamamoto K, Yuyama K, Nakamura H, et al Extracellular vesicles from amnion-derived mesenchymal stem cells ameliorate hepatic inflammation and fibrosis in rats. Stem Cells Int. 2018;2018.

25. Bruno S, Pasquino C, Sanchez MBH, Tapparo M, Figliolini F, Grange C, et al. HLSC-derived extracellular vesicles attenuate liver fibrosis and inflammation in a murine model of non-alcoholic steatohepatitis. Mol Ther. 2020;28(2):479-89.

26. Zhu F, Shin OLSCL, Pei G, Hu Z, Yang J, Zhu H, et al. Adipose-derived mesenchymal stem cells employed exosomes to attenuate AKI-CKD transition through tubular epithelial cell dependent Sox9 activation. Oncotarget. 2017;8(41):70707-70726.

27. Zhong L, Liao G, Wang X, Li L, Zhang J, Chen Y, et al. Mesenchymal stem cells-microvesicle-miR-451a ameliorate early diabetic kidney injury by negative regulation of P15 and P19. Exp Biol Med. 2018;243(15-16):1233-42.

28. Grange C, Tritta S, Tapparo M, Cedrino M, Tetta C, Camussi G, et al. Stem cell-derived extracellular vesicles inhibit and revert fibrosis progression in a mouse model of diabetic nephropathy. Sci Rep. 2019;9.

29. Kholia S, Herrera Sanchez MB, Cedrino M, Papadimitriou E, Tapparo M, Deregibus MC, et al. Mesenchymal stem cell derived extracellular vesicles ameliorate kidney injury in aristolochic acid nephropathy. Front Cell Dev Biol. 2020;8:188-188.

30. Jin J, Wang Y, Zhao L, Zou W, Tan M, He Q. Exosomal miRNA-215-5p derived from adipose-derived stem cells attenuates epithelial-mesenchymal transition of podocytes by inhibiting ZEB2. Biomed Res Int. 2020;2020.

31. Wang B, Yao K, Huuskes BM, Shen H-H, Zhuang J, Godson C, et al. Mesenchymal stem cells deliver exogenous microRNA-let7c via exosomes to attenuate renal fibrosis. Mol Ther. 2016;24(7):1290-301.

32. Ji C, Zhang J, Zhu Y, Shi H, Yin S, Sun F, et al. Exosomes derived from huCMSC attenuate renal fibrosis through CK1 delta/beta-TRCP-mediated YAP degradation. Cell Death Disease. 2020;11(5).

33. Liu B, Hu D, Zhou Y, Yu Y, Shen L, Long C, et al. Exosomes released by human umbilical cord mesenchymal stem cells protect against renal interstitial fibrosis through ROS-mediated P38MAPK/ERK signaling pathway. Am J Transl Res. 2020;12(9):4998-5014.

34. Shi Z, Wang Q, Zhang Y, Jiang D. Extracellular vesicles produced by bone marrow mesenchymal stem cells attenuate renal fibrosis, in part by inhibiting the RhoA/ROCK pathway, in a UUO rat model. Stem Cell Res Therapy. 2020;11(1).

35. Chen F, Li X, Zhao J, Geng J, Xie J, Xu B. Bone marrow mesenchymal stem cell-derived exosomes attenuate cardiac hypertrophy and fibrosis 
in pressure overload induced remodeling. Vitro Cell Dev Biol Animal. 2020;56(7):567-76.

36. Wang S, Li L, Liu T, Jiang W, Hu X. miR-19a/19b-loaded exosomes in combination with mesenchymal stem cell transplantation in a preclinical model of myocardial infarction. Regen Med. 2020;15(6):1749-59.

37. Deng S, Zhou X, Ge Z, Song Y, Wang H, Liu X, et al. Exosomes from adipose-derived mesenchymal stem cells ameliorate cardiac damage after myocardial infarction by activating S1P/SK1/S1PR1 signaling and promoting macrophage M2 polarization. Int J Biochem Cell Biol. 2019;114.

38. Wang X, Zhu Y, Wu C, Liu W, He Y, Yang Q. Adipose-derived mesenchymal stem cells-derived exosomes carry microRNA-671 to alleviate myocardial infarction through inactivating the TGFBR2/Smad2 Axis. Inflammation. 2021.

39. Fang $\mathrm{S}$, Xu C, Zhang Y, Xue C, Yang C, Bi H, et al. Umbilical cord-derived mesenchymal stem cell-derived exosomal MicroRNAs suppress myofibroblast differentiation by inhibiting the transforming growth factorbeta/SMAD2 pathway during wound healing. Stem Cells Transl Med. 2016:5(10):1425-39.

40. Wang L, Hu L, Zhou X, Xiong Z, Zhang C, Shehada HMA, et al. Exosomes secreted by human adipose mesenchymal stem cells promote scarless cutaneous repair by regulating extracellular matrix remodelling. Sci Rep. 2017;7.

41. Dalirfardouei R, Jamialahmadi K, Jafarian AH, Mahdipour E. Promising effects of exosomes isolated from menstrual blood-derived mesenchymal stem cell on wound-healing process in diabetic mouse model. J Tissue Eng Regen Med. 2019;13(4):555-68.

42. Li Y, Zhang J, Shi J, Liu K, Wang X, Jia Y, et al. Exosomes derived from human adipose mesenchymal stem cells attenuate hypertrophic scar fibrosis by miR-192-5p/L-17RA/Smad axis. Stem Cell Res Therapy. 2021;12(1).

43. Guo L, Lai P, Wang Y, Huang T, Chen X, Geng S, et al. Extracellular vesicles derived from mesenchymal stem cells prevent skin fibrosis in the CGVHD mouse model by suppressing the activation of macrophages and B cells immune response. Int Immunopharmacol. 2020;84.

44. Tan Q, Xia D, Ying X. miR-29a in exosomes from bone marrow mesenchymal stem cells inhibit fibrosis during endometrial repair of intrauterine adhesion. Int J Stem Cells. 2020;13(3):414-23.

45. Xiao B, Zhu Y, Huang J, Wang T, Wang F, Sun S. Exosomal transfer of bone marrow mesenchymal stem cell-derived miR-340 attenuates endometrial fibrosis. Biology Open. 2019;8(5).

46. Yao Y, Chen R, Wang G, Zhang Y, Liu F. Exosomes derived from mesenchymal stem cells reverse EMT via TGF-beta 1/Smad pathway and promote repair of damaged endometrium. Stem Cell Res Therapy. 2019;10.

47. Yao Z, Li J, Wang X, Peng S, Ning J, Qian Y, et al. MicroRNA-21-3p engineered umbilical cord stem cell-derived exosomes inhibit tendon adhesion. J Inflamm Res. 2020;13:303-16.

48. Yang J, Zhou CZ, Zhu R, Fan H, Liu XX, Duan XY, et al. miR-200b-containing microvesicles attenuate experimental colitis associated intestinal fibrosis by inhibiting epithelial-mesenchymal transition. J Gastroenterol Hepatol. 2017;32(12):1966-74.

49. Wijsenbeek M, Cottin V. Spectrum of fibrotic lung diseases. N Engl J Med. 2020;383(10):958-68.

50. Zhang C, Yin X, Zhang J, Ao Q, Gu Y, Liu Y. Clinical observation of umbilical cord mesenchymal stem cell treatment of severe idiopathic pulmonary fibrosis: a case report. Exp Ther Med. 2017;13(5):1922-6.

51. Gentile P. SARS-CoV-2: the "uncensored" truth about its origin and adipose-derived mesenchymal stem cells as new potential immunemodulatory weapon. Aging Dis. 2021;12(2):330-44.

52. Gentile P, Sterodimas A. Adipose stem cells (ASCs) and stromal vascular fraction (SVF) as a potential therapy in combating (COVID-19)-disease. Aging Dis. 2020;11(3):465-9.

53. Chaubey S, Thueson S, Ponnalagu D, Alam MA, Gheorghe CP, Aghai Z, et al. Early gestational mesenchymal stem cell secretome attenuates experimental bronchopulmonary dysplasia in part via exosome-associated factor TSG-6. Stem Cell Res Therapy. 2018;9.

54. Phinney DG, Di Giuseppe M, Njah J, Sala E, Shiva S, St Croix CM, et al. Mesenchymal stem cells use extracellular vesicles to outsource mitophagy and shuttle microRNAs. Nat Commun. 2015;6.

55. Lou G, Yang Y, Liu F, Ye B, Chen Z, Zheng M, et al. MiR-122 modification enhances the therapeutic efficacy of adipose tissue-derived mesenchymal stem cells against liver fibrosis. J Cell Mol Med. 2017;21(11):2963-73.
56. Qu Y, Zhang Q, Cai X, Li F, Ma Z, Xu M, et al. Exosomes derived from miR181-5p-modified adipose-derived mesenchymal stem cells prevent liver fibrosis via autophagy activation. J Cell Mol Med. 2017;21(10):2491-502.

57. Zhu M, Liu X, Li W, Wang L. Exosomes derived from mmu_circ_0000623modified ADSCs prevent liver fibrosis via activating autophagy. Hum Exp Toxicol. 2020;39(12):1619-27.

58. Mardpour S, Ghanian MH, Sadeghi-Abandansari H, Mardpour S, Nazari A, Shekari F, et al. Hydrogel-mediated sustained systemic delivery of mesenchymal stem cell-derived extracellular vesicles improves hepatic regeneration in chronic liver failure. ACS Appl Mater Interfaces. 2019;11(41):37421-33.

59. Takeuchi S, Tsuchiya A, Iwasawa T, Nojiri S, Watanabe T, Ogawa M, et al. Small extracellular vesicles derived from interferon-gamma pre-conditioned mesenchymal stromal cells effectively treat liver fibrosis. NPJ Regener Med. 2021;6(1):19-19.

60. Nastase MV, Zeng-Brouwers J, Wygrecka M, Schaefer L. Targeting renal fibrosis: mechanisms and drug delivery systems. Adv Drug Deliv Rev. 2018;129:295-307.

61. Zhou Y, Liu S, Zhao M, Wang C, Li L, Yuan Y, et al. Injectable extracelIular vesicle-released self-assembling peptide nanofiber hydrogel as an enhanced cell-free therapy for tissue regeneration. J Control Release. 2019;316:93-104.

62. Chen L, Wang Y, Li S, Zuo B, Zhang X, Wang F, et al. Exosomes derived from GDNF-modified human adipose mesenchymal stem cells ameliorate peritubular capillary loss in tubulointerstitial fibrosis by activating the SIRT1/eNOS signaling pathway. Theranostics. 2020;10(20):9425-42.

63. Zhang C, Shang Y, Chen X, Midgley AC, Wang Z, Zhu D, et al. Supramolecular nanofibers containing arginine-glycine-aspartate (RGD) peptides boost therapeutic efficacy of extracellular vesicles in kidney repair. ACS Nano. 2020;14(9):12133-47.

64. Liu Y, Cui J, Wang H, Hezam K, Zhao X, Huang H, et al. Enhanced therapeutic effects of MSC-derived extracellular vesicles with an injectable collagen matrix for experimental acute kidney injury treatment. Stem Cell Res Therapy. 2020;11(1).

65. Zhang Z-Y, Hou Y-P, Zou X-Y, Xing X-Y, Ju G-Q, Zhong L, et al. Enhanced the therapeutic effects of mesenchymal stem cell-derived extracellular vesicles in acute kidney injury. Kidney Blood Press Res. 2020;45(1):95-108.

66. Anderson JL, Morrow DA. Acute myocardial infarction. N Engl J Med. 2017;376(21):2053-64.

67. Ferguson SW, Wang J, Lee CJ, Liu M, Neelamegham S, Canty JM, et al. The microRNA regulatory landscape of MSC-derived exosomes: a systems view. Sci Rep. 2018;8.

68. Han C, Zhou J, Liang C, Liu B, Pan X, Zhang Y, et al. Human umbilical cord mesenchymal stem cell derived exosomes encapsulated in functional peptide hydrogels promote cardiac repair. Biomater Sci. 2019;7(7):2920-33.

69. Lv K, Li Q, Zhang L, Wang Y, Zhong Z, Zhao J, et al. Incorporation of small extracellular vesicles in sodium alginate hydrogel as a novel therapeutic strategy for myocardial infarction. Theranostics. 2019;9(24):7403-16.

70. Firoozi S, Pahlavan S, Ghanian M-H, Rabbani S, Barekat M, Nazari A, et al. Mesenchymal stem cell-derived extracellular vesicles alone or in conjunction with a SDKP-conjugated self-assembling peptide improve a rat model of myocardial infarction. Biochem Biophys Res Commun. 2020;524(4):903-9.

71. Pan J, Alimujiang M, Chen Q, Shi H, Luo X. Exosomes derived from miR146a-modified adipose-derived stem cells attenuate acute myocardial infarction-induced myocardial damage via downregulation of early growth response factor 1. J Cell Biochem. 2019;120(3):4433-43.

72. Wang $X$, Chen $Y$, Zhao Z, Meng Q, Yu Y, Sun J, et al. Engineered exosomes with ischemic myocardium-targeting peptide for targeted therapy in myocardial infarction. J Am Heart Assoc. 2018;7(15).

73. Deng J, Zhang N, Wang Y, Yang C, Wang Y, Xin C, et al. FNDC5/irisin improves the therapeutic efficacy of bone marrow-derived mesenchymal stem cells for myocardial infarction. Stem Cell Res Therapy. 2020;11(1).

74. Sun J, Shen H, Shao L, Teng X, Chen Y, Liu X, et al. HIF-1 alpha overexpression in mesenchymal stem cell-derived exosomes mediates cardioprotection in myocardial infarction by enhanced angiogenesis. Stem Cell Res Therapy. 2020;11(1).

75. Shao L, Zhang Y, Pan X, Liu B, Liang C, Zhang Y, et al. Knockout of beta-2 microglobulin enhances cardiac repair by modulating exosome 
imprinting and inhibiting stem cell-induced immune rejection. Cell Mol Life Sci. 2020;77(5):937-52.

76. Feng Y, Huang W, Wani M, Yu X, Ashraf M. Ischemic preconditioning potentiates the protective effect of stem cells through secretion of exosomes by targeting Mecp2 via miR-22. Plos One. 2014;9(2).

77. Zhu J, Lu K, Zhang N, Zhao Y, Ma Q, Shen J, et al. Myocardial reparative functions of exosomes from mesenchymal stem cells are enhanced by hypoxia treatment of the cells via transferring microRNA-210 in an nSMase2-dependent way. Artif Cells Nanomed Biotechnol. 2018;46(8):1659-70.

78. Jiang L, Zhang Y, Liu T, Wang X, Wang H, Song $\mathrm{H}$, et al. Exosomes derived from TSG-6 modified mesenchymal stromal cells attenuate scar formation during wound healing. Biochimie. 2020;177:40-9.

79. Shen Y, Xu G, Huang H, Wang K, Wang H, Lang M, et al. Sequential release of small extracellular vesicles from bilayered thiolated alginate/polyethylene glycol diacrylate hydrogels for scarless wound healing. ACS nano. 2021.

80. Li J, Yao Z, Xiong H, Cui H, Wang X, Zheng W, et al. Extracellular vesicles from hydroxycamptothecin primed umbilical cord stem cells enhance anti-adhesion potential for treatment of tendon injury. Stem Cell Res Therapy. 2020;11(1)

81. Liang Y-C, Wu Y-P, Li X-D, Chen S-H, Ye X-J, Xue X-Y, et al. TNFalpha-induced exosomal miR-146a mediates mesenchymal stem cell-dependent suppression of urethral stricture. J Cell Physiol. 2019;234(12):23243-55

82. Kou L, Jiang X, Xiao S, Zhao Y-Z, Yao Q, Chen R. Therapeutic options and drug delivery strategies for the prevention of intrauterine adhesions. J Control Release. 2020;318:25-37.
83. Zhao Y, Zhu X, Zhang L, Ferguson CM, Song T, Jiang K, et al. Mesenchymal stem/stromal cells and their extracellular vesicle progeny decrease injury in poststenotic swine kidney through different mechanisms. Stem Cells Dev. 2020;29(18):1190-200

84. Eirin A, Zhu XY, Jonnada S, Lerman A, van Wijnen AJ, Lerman LO. Mesenchymal stem cell-derived extracellular vesicles improve the renal microvasculature in metabolic renovascular disease in swine. Cell Transplant. 2018;27(7):1080-95.

85. Zhang L, Zhu XY, Zhao Y, Eirin A, Liu L, Ferguson CM, et al. Selective intrarenal delivery of mesenchymal stem cell-derived extracellular vesicles attenuates myocardial injury in experimental metabolic renovascular disease. Basic Res Cardiol. 2020;115(2):16.

86. Li D, Lu Z, Li X, Xu Z, Jiang J, Zheng Z, et al. Human umbilical cord mesenchymal stem cells facilitate the up-regulation of miR-153-3p, whereby attenuating MGO-induced peritoneal fibrosis in rats. J Cell Mol Med. 2018;22(7):3452-63.

87. Shekari F, Nazari A, Assar Kashani S, Hajizadeh-Saffar E, Lim R, Baharvand $\mathrm{H}$. Pre-clinical investigation of mesenchymal stromal cell-derived extracellular vesicles: a systematic review. Cytotherapy. 2021;23(4):277-84.

88. Caplan H, Olson SD, Kumar A, George M, Prabhakara KS, Wenzel P, et al. Mesenchymal stromal cell therapeutic delivery: translational challenges to clinical application. Front Immunol. 2019;10.

\section{Publisher's Note}

Springer Nature remains neutral with regard to jurisdictional claims in published maps and institutional affiliations.
Ready to submit your research? Choose BMC and benefit from:

- fast, convenient online submission

- thorough peer review by experienced researchers in your field

- rapid publication on acceptance

- support for research data, including large and complex data types

- gold Open Access which fosters wider collaboration and increased citations

- maximum visibility for your research: over 100M website views per year

At BMC, research is always in progress.

Learn more biomedcentral.com/submissions 